\title{
Ion beam figuring and optical metrology system for synchrotron $\mathrm{x}$ - ray mirrors
}

Matthew Hand, Simon Alcock, Michael Hillman, Richard Littlewood, Simone Moriconi, et al.

Matthew Hand, Simon G. Alcock, Michael Hillman, Richard Littlewood, Simone Moriconi, Hongchang Wang, Kawal Sawhney, "Ion beam figuring and optical metrology system for synchrotron x-ray mirrors," Proc. SPIE 11109, Advances in Metrology for X-Ray and EUV Optics VIII, 111090A (9 September 2019); doi: 10.1117/12.2528463

SPIE Event: SPIE Optical Engineering + Applications, 2019, San Diego, California, United States 


\title{
Ion Beam Figuring and Optical Metrology System for Synchrotron X-ray Optics
}

\author{
Matthew Hand*a, Simon G. Alcock ${ }^{\mathrm{a}}$, Michael Hillman ${ }^{\mathrm{a}}$, Richard Littlewood ${ }^{\mathrm{a}}$, Simone Moriconi ${ }^{\mathrm{a}}$, \\ Hongchang Wang ${ }^{a}$, Kawal Sawhney ${ }^{\mathrm{a}}$ \\ a Diamond Light Source, Harwell Science \& Innovation Campus, Didcot, OX11 0DE, UK
}

\begin{abstract}
Meeting the ever-increasing performance demands of X-ray beamlines at modern synchrotrons, such as Diamond Light Source (DLS), requires the use of ultra-high-quality X-ray mirrors with surface deviations of less than a few nanometres from their ideal shape. Ion beam figuring (IBF) is frequently used for creating mirrors of this precision, but achieving the highest accuracy is critically dependent on careful alignment and precise metrology of defects on the optical surface. Multiple iterations of measurement and correction are typically required, and convergence towards the requisite shape can be a slow process. DLS have designed and built an in-house IBF system that comprises a large diameter DC gridded ion source, and a 4-axis motion stage for manipulating the mirror being figured. Additionally, a slope measuring profilometer for in-situ metrology, and an imaging system for alignment, are also built into the system. The advantages of incorporating these extra components are twofold: fast metrology feedback after each figuring run will considerably reduce the time required to perform multiple figuring iterations; and alignment and indexing errors will be drastically reduced when transferring the optic. Complemented by the Optical Metrology Laboratory at DLS and at-wavelength X-ray measurements on the Test beamline B16, it is expected that this system will enable rapid development and testing of high-quality mirrors with novel designs for micro- and nano-focussing of X-rays.
\end{abstract}

Keywords: ion beam figuring, X-ray optics, synchrotron, optical metrology

*matthew.hand@diamond.ac.uk

\section{INTRODUCTION}

Beamlines at Diamond Light Source (DLS) utilize a wide variety of X-ray optics with different shapes and sizes, but a common theme is the requirement of an ultra-high-quality finish on the optically active surfaces. Surface deviations from the ideal profile (so called height or figure errors) often need to be below $1 \mathrm{~nm}$ rms to achieve some of the most demanding $\mathrm{X}$-ray applications including nano-focusing, preserve coherence, or extreme energy resolution. This level of surface quality for non-planar optics cannot easily be achieved using traditional mechanical polishing techniques. This motivates the use of advanced deterministic polishing methods, such as ion beam figuring ${ }^{1,2}$, for the final stages of optic production. Such specialized tools are possessed by only a few suppliers of X-ray optics for synchrotron and free electron laser beamlines, which presents a significant procurement cost and time risk.

High-precision metrology feedback is often the limiting factor for achieving the ultimate quality optics using deterministic correction techniques. The Optical Metrology Lab at Diamond ${ }^{3}(\mathrm{OML})$ is an ultra-stable laboratory equipped with a suite of metrology instruments which exceeds the capabilities of many manufacturers. Previously, the OML have collaborated with optical fabricators by supplying metrology data to be used as the input for ion beam correction of surface defects. The optics were significantly improved by the process, by a factor $>2$ in many cases, and slope errors of $<100$ nanoradians rms were achieved ${ }^{4}$. However, multiple cycles of handling and transportation back and forth between the supplier and DLS greatly increases the risk of damage to the optics. It is also time consuming: approx. 1 month was required for each IBF iteration, and 3-4 cycles were required for correction of some mirrors.

Since one half of the IBF equation - state-of-the-art metrology - is already available at DLS, it is natural to consider implementation of the other half at DLS to create ultra-high-quality X-ray optics. This second step has already been taken at several other synchrotron facilities around the world ${ }^{5-7}$. Purchasing a turn-key system was considered, but quickly rejected due to high costs and lack of flexibility for future development. Since DLS also possesses a great deal of technical expertise (mechanical and electrical engineers, motion control engineers, software developers, etc.) it was a sensible option 
to design and build our own ion beam figuring system. Doing so has provided several key advantages: novel features notably, an on-board metrology system - have been incorporated; the system has been designed such that it can be readily transported to the B16 Test beamline for combined at-wavelength X-ray measurement ${ }^{8,9}$ and IBF processing; and the whole system has been built to DLS motion controls, electrical and I.T. standards which allows for easy integration with existing infrastructure and makes it much more straightforward for technical support and development of the system.

\section{SYSTEM SPECIFICATIONS}

\subsection{Overview}

The IBF system primarily consists of a large vacuum vessel with dimensions $1.6 \mathrm{~m} \times 0.9 \mathrm{~m} \times 1.0 \mathrm{~m}$ (approx.) which is connected to a steel support structure. Large, hinged doors at both ends of the vessel provide clear access for workpiece loading and maintenance of in-vacuum components. Evacuation of the chamber is achieved by a nEXT400D turbomolecular pump mounted on the vessel, and an nXDS20i dry scroll pump. The scroll pump, which backs the turbo pump, is purposefully located outside of the lab, in the main experimental hall, to minimize noise and vibrations inside the lab. This pump combination is designed to provide a base pressure of $\sim 1 \times 10^{-6} \mathrm{mbar}$. The other main components mounted on the outside of the vacuum vessel, as shown in Fig. 1a, are the ion source, autocollimator support, and CCD camera. All components are described in detail below. The main components inside the chamber, as shown in Figure 1(b), are the invacuum motion stages which moves the optic being shaped (workpiece) in front of the ion beam during the figuring process, and a set of apertures that are used to define the final size and shape of the ion beam. These assemblies are mounted onto an aluminium breadboard that can removed from the chamber on a slide rail system should major maintenance or modification work be required.

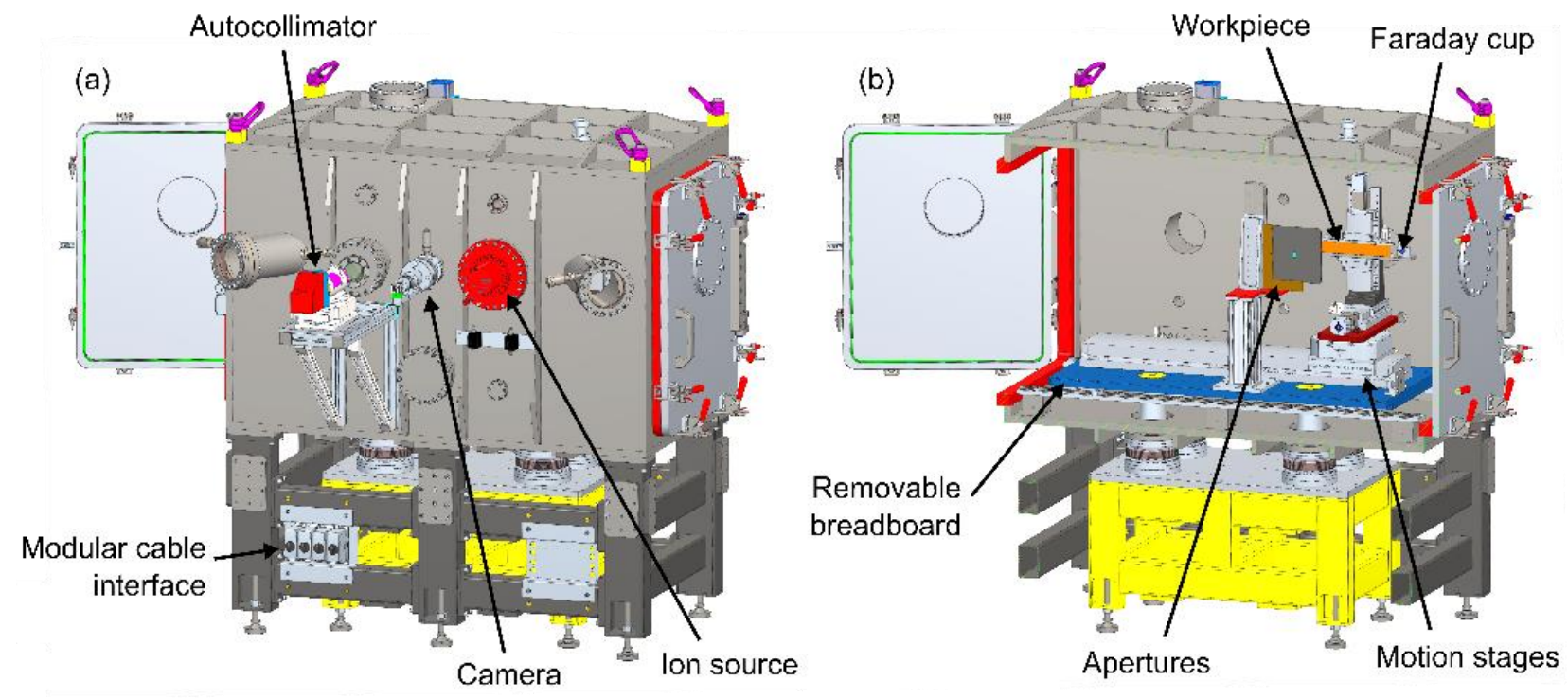

Figure 1: 3D model of IBF system designed and built at DLS. (a) Ion source, camera, and autocollimator are mounted externally on the vacuum vessel. (b) In-vacuum components, such as the motion stages and apertures, are mounted on a removable breadboard (highlighted in blue).

\subsection{Ion Source}

A Kaufman \& Robinson (KRi) KDC100 ion source, shown in Figure 2(a), generates the ion beam to be used for the IBF process. It is equipped with dished divergent grid ion optics and emits a circular shaped ion beam with a diameter of $12 \mathrm{~cm}$. The ion source is fed with argon (Ar) gas (99.999+\% purity) via a mass flow controller (MFC), shown in Figure 2(b), that can provide flow rates up to $50 \mathrm{sccm}$ (standard cubic centimeters per minute). An external neutralizer, mounted onto the ion source, injects electrons into the main ion beam for space-charge compensation. The neutralizer is also fed with Ar gas and utilizes its own independent MFC. This combination of ion source and slightly divergent optics generates a circular 
ion beam with a large central portion of uniform current density. A summary of the specifications of the ion source is listed in Table 1.

Table 1: Summary of the specifications of the ion source.

\begin{tabular}{|l|l|}
\hline Manufacturer & Kaufman \& Robinson (KRi) \\
\hline Model & KDC100 \\
\hline Source type & DC thermionic discharge \\
\hline Diameter of ion beam & $12 \mathrm{~cm}$ \\
\hline Ion optics & Gridded, dished divergent \\
\hline Ion beam energy & $100-1200 \mathrm{~V}$ \\
\hline Maximum ion beam current & $>400 \mathrm{~mA}$ \\
\hline Operating gas & $\mathrm{Ar}$ \\
\hline Typical gas flow rate & $2-20 \mathrm{sccm}$ \\
\hline Neutralizer & KRi LFN2000 (external) \\
\hline Operational pressure & $\sim 3 \times 10^{-4} \mathrm{mbar}$ \\
\hline
\end{tabular}

Two sets of apertures, manufactured from pyrolytic graphite due to its mechanical resistance to ion sputtering, define the size and shape of the ion beam. Firstly, a large plate allows only the central $20 \mathrm{~mm} \times 10 \mathrm{~mm}$ portion of the ion beam through. A second plate, downstream of the first, contains several apertures of different shapes and dimensions which allow the user to define the final size of the ion beam incident upon the test mirror. This second aperture plate is mounted on a linear translation stage to move between the different apertures.
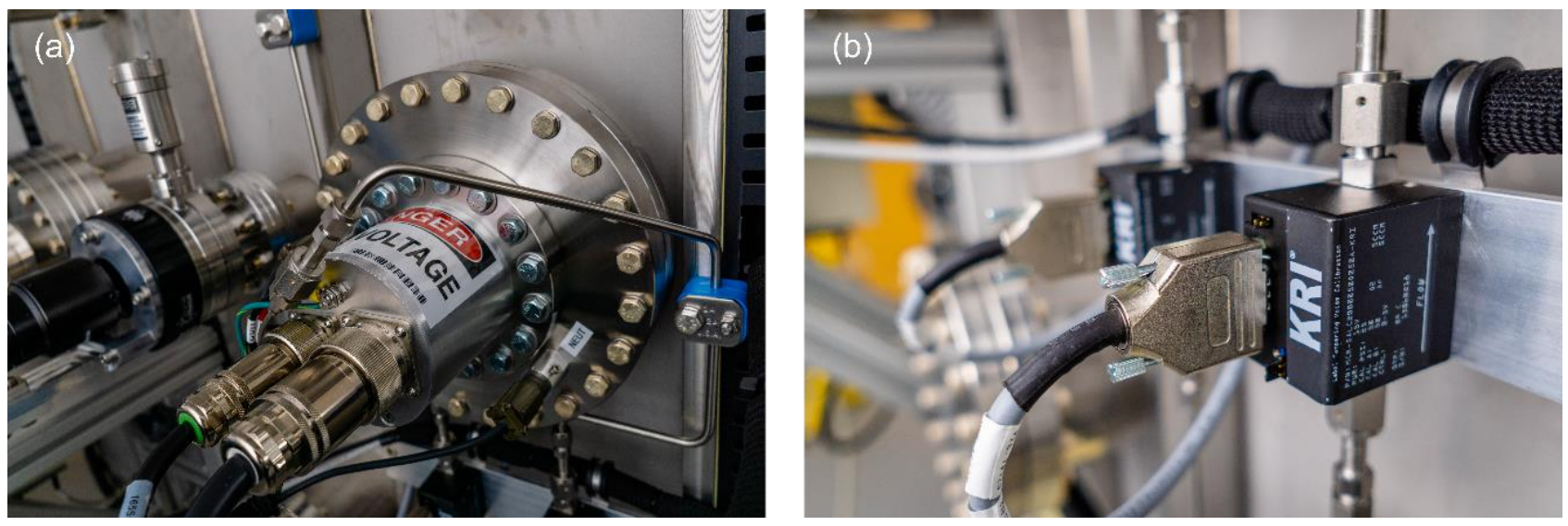

Figure 2: (a) The Ar ion beam is generated by a KRi KDC100 ion source. Apertures are used to select the size and shape of the ion beam incident upon the mirror being figured. (b) The flow rate of Ar gas supplied to the source and neutralizer can be set independently via mass flow controllers.

\subsection{Motion System}

The test mirror / workpiece is mounted on a set of in-vacuum motion stages (procured from Aerotech) which provide 4degrees of freedom $(3 \times$ translation +1 rotation). The test mirror is oriented with its surface normal pointing in the horizontal direction. For this IBF system, the mirror moves, whilst the ion beam gun stays fixed. The holder can accommodate optics up to $300 \mathrm{~mm}$ in length. A PRO225LM linear stage with a brushless servo motor and a travel range of $800 \mathrm{~mm}$ is the primary axis for controlling the position of the workpiece during an IBF run. Importantly, the servo motor 
driving the motion stage allows for the velocity and acceleration to be changed dynamically for precise control of the dwell time as the workpiece is exposed to the ion beam.

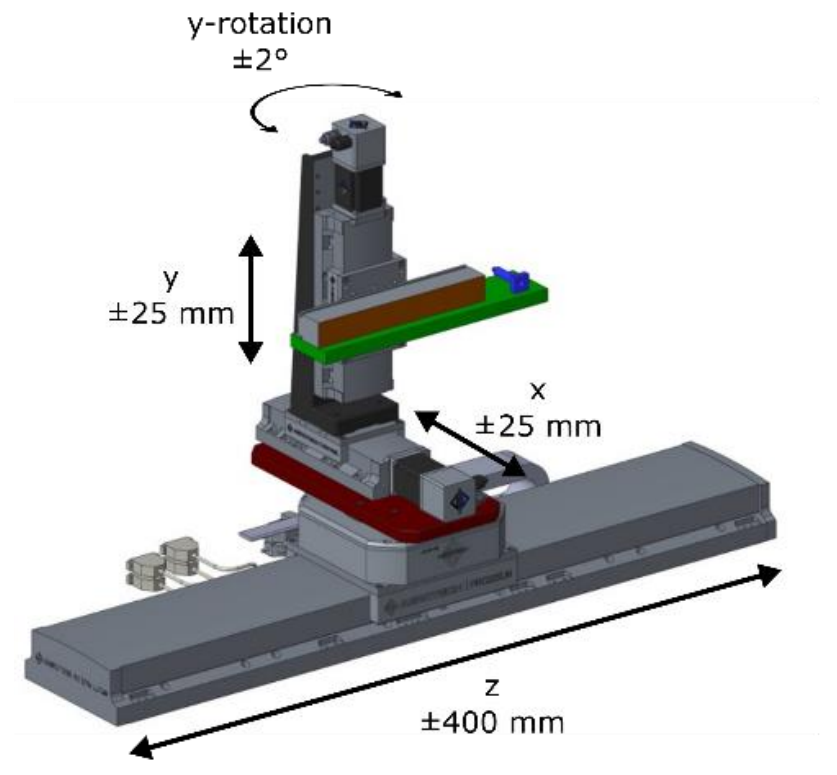

Figure 3: A 4-axis in-vacuum motion stage system is used to manipulate the workpiece.

Vertical movement and motion along the ion beam's axis are provided by two orthogonally mounted PRO115SLE linear stages, each with a $50 \mathrm{~mm}$ travel range and driven by stepper motors. An ALAR100LP rotary stage allows pitch adjustment of the workpiece by up to $4^{\circ}$. All axes are encoded and operate in closed-loop control. Finally, an FC-66 Faraday cup (purchased from Kimball Physics) is also mounted at the end of the workpiece holder. The Faraday cup detector can be scanned in front of the ion source to determine the current and spatial profile of the ion beam.

\subsection{Metrology}

Inclusion of on-board metrology capability is a key feature of this IBF system. An Elcomat3000 autocollimator (AC) from Möller-Wedel, shown in Figure 4(a), will be used to measure the slope profile of the test optic through one of the viewports, as the motion stage translates it past the $\mathrm{AC}$ beam. The viewport contains a high-quality fused silica window, which minimizes disturbances to the $\mathrm{AC}$ signal, and is purposefully tilted to eliminate unwanted specular reflection entering the AC. As part of the commissioning process, the motion stage system will be characterized to measure residual parasitic pitch motions. The effect of these systematic errors can then be removed from subsequent slope profile measurements to more accurately reveal the shape of the test mirror.

In the early stages of operation, optical metrology will be performed in the OML and slope profiles measured using the Diamond-NOM. This will provide the primary input into dwell time calculations of material to be removed from the test mirror. Accurate calibration between the coordinate systems of the NOM and the IBF system is essential. This will be achieved using a fiducialization procedure to index the location of reference markers on the optic when it is transferred between the two instruments. A Mako-G CCD camera (Allied Vision) fitted with a Thorlabs 18-108 mm zoom lens (up to 130x magnification), shown in Figure 4(b), will be used to observe the fiducials to aid accurately alignment of the workpiece. In addition to providing inputs for the dwell-time calculation, data from the Diamond-NOM will also help to understand and ultimately reduce the systematic measurement errors of the onboard metrology system. 

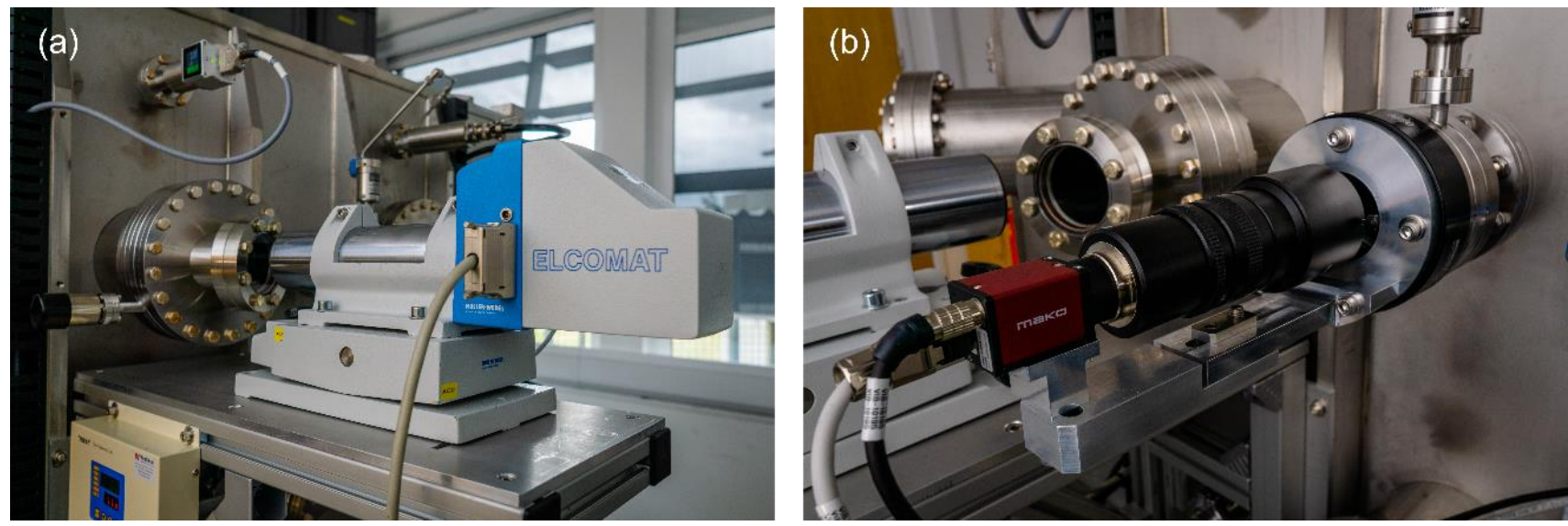

Figure 4: (a) An Elcomat3000 autocollimator is mounted to the system for in-situ metrology of the test mirror. (b) A Mako-G camera system will be used for alignment of the workpiece relative to the position of the ion beam.

\subsection{Control System}

Operation of the various sub-components of the IBF system is controlled via the hardware rack, shown in Figure 5. A set of modular cables connects the rack to the main IBF system, which greatly simplifies transportation between the lab and the beamline. A touchscreen human-machine interface (HMI) is used to control the vacuum system. A programmable logic controller (PLC) ensures that pumping and venting are executed in a safe manner. The PLC also handles interlocks for the motion stages and ion source for operator safety and machine protection. Motion control is provided by Diamond's standard motion controller, a Geo Brick LV (Delta Tau), which supports position-velocity-time (PVT) control of the long linear stage. A set of KRi ion source controller modules operates the ion source and neutralizer and allows for local and remote control of all electrical and gas flow parameters. The main user interface for operation of the IBF system is the integrated workstation computer that also acts a server to run the EPICS input/output controllers (IOCs). This allows for programmatic control of the major subsystems (ion source, motion, vacuum) and will be the foundation for a future software control suite.
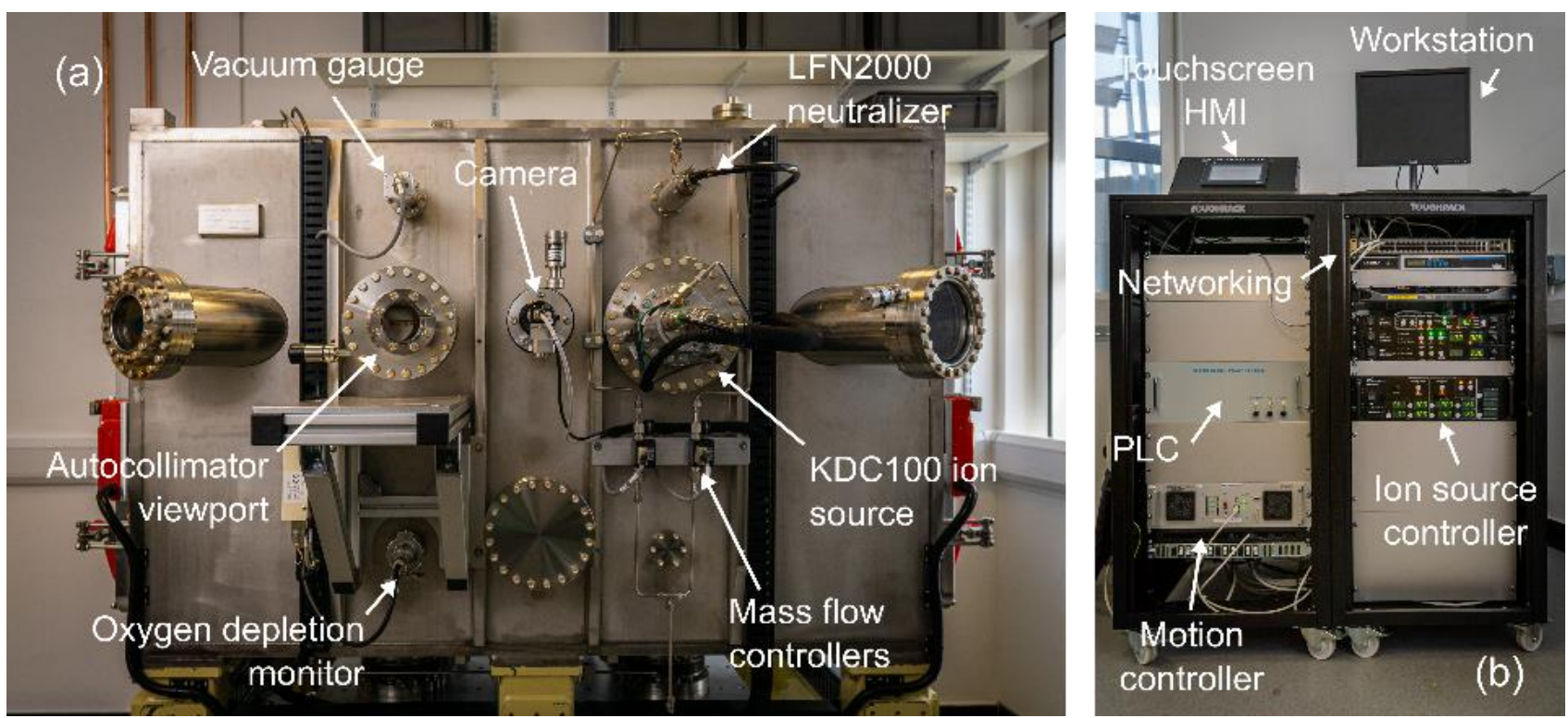

Figure 5: Photographs of (a) the IBF vacuum vessel and (b) control rack in the IBF lab at DLS. The vessel and the rack are connected by a set of modular cables which allows for easy connection and disconnection when the system is transported. 


\section{FURTHER WORK}

Following completion of the build phase and successful first ignition of the ion source in Summer 2019, the primary project objective will be to conclude full commissioning of all sub-systems. This will include: fine tuning of the motion stages for optimal dynamic performance; determining suitable parameters for stable operation of the ion source; and calibrating the Faraday cup detector to accurately measure the ion beam current.

The goal for the next few months is to test dwell-time algorithms and begin to use the ion beam to deterministically modify the surface of test optics, initially by removing material in the form of simple mathematical functions. This will require complete characterization of the ion source, for a range of different conditions, to find the most suitable parameters. Material removal rates will be determined by making several, static exposures for different dwell times at multiple locations on a super-smooth silicon test wafer. The depth of the resultant ion beam "craters" will be imaged using a Bruker Contour GT-X micro-interferometer in the OML. Surface micro-roughness of these wafers is purposefully comparable to that of a typical synchrotron X-ray mirror $(<0.3 \mathrm{~nm} \mathrm{rms})$. Therefore, high magnification (e.g. 50x) measurements at the base of the craters will also determine whether micro-roughness degradation has occurred. If so, the IBF process configuration will be adjusted to correct this. The size and shape of the ion beam can be measured using 2D scans of the Faraday cup detector. Using this knowledge, it will then be possible to calculate the ion beam removal functions which provide inputs into the dwell-time calculation.

Figuring of basic quality silicon mirrors will then be attempted. This will include improving their flatness or producing sinusoids or chirped profiles along the mirror's surface. Alongside the initial figuring tests, research will be also be undertaken to perfect the fiducialization procedure for precise alignment $(<100 \mu \mathrm{m})$ between the coordinate axes of the IBF system and the metrology instruments in the OML. Additionally, tests of the onboard metrology system will be carried out. If successful, this will allow for complete onboard measurement-figuring iterations to begin.

Once the IBF system is fully operational and the figuring procedure is well-established, we will then look towards moving the system to the B16 Test beamline to utilize X-ray metrology, optical metrology, and ion beam figuring to produce stateof-the-art and novel X-ray optics.

\section{CONCLUSIONS}

An ion beam figuring system has been designed and built at Diamond Light Source. Initial testing is now underway following successful commissioning of all individual sub-systems and safety features. In-house design means that each sub-system is fully configurable. The result is an ideal platform for research and development of ion beam correction to create ultra-high-quality synchrotron X-ray optics. By utilizing the cutting-edge metrology capabilities at DLS it is expected that the IBF system can ultimately create state-of-the-art and novel X-ray mirrors with slope errors below $100 \mathrm{nrad}$ rms. Planning has already begun for the Diamond-II machine upgrade which will occur in the next few years ${ }^{10}$. This will lead to demands for further improvements to the quality of X-ray optics. It is hoped that in-house optical finishing capabilities will produce improved quality X-ray mirrors which will take full advantage of the upgraded light source.

\section{ACKNOWLEDGEMENTS}

This work was carried out with the support of Diamond Light Source Ltd. (UK). The authors would like to thank many staff at Diamond for their invaluable contributions to the design and building of this system, including Andrew Malandain, Russel Marshall, Ben Garvey, Harriott Nowell, Enrique Rodriguez-Garcia, Paul Amos, Nico Rubies, Emilio Perez-Juarez, Hugo Shiers, Simon Lay and Richard Lear.

\section{REFERENCES}

[1] Thiess, H., Lasser, H. and Siewert, F., "Fabrication of X-ray mirrors for synchrotron applications,” Nucl. Instrum. Methods Phys. Res. A 616(2-3), 157-161 (2010).

[2] Arnold, T. and Pietag, F., "Ion beam figuring machine for ultra-precision silicon spheres correction," Precis. Eng. 41, 119-125 (2015).

[3] Alcock, S. G., Sawhney, K. J. S., Scott, S., Pedersen, U., Walton, R., Siewert, F., Zeschke, T., Senf, F., Noll, T. and Lammert, H., "The Diamond-NOM: A non-contact profiler capable of characterizing optical figure error 
with sub-nanometre repeatability," Nucl. Instrum. Methods Phys. Res. A 616(2-3), 224-228 (2010).

[4] Alcock, S. G., Nistea, I. and Sawhney, K., "Nano-metrology: The art of measuring X-ray mirrors with slope errors <100 nrad," Rev. Sci. Instrum. 87(5) (2016).

[5] Peverini, L., Kozhevnikov, I. V., Rommeveaux, A., Vaerenbergh, P. V., Claustre, L., Guillet, S., Massonnat, J.Y., Ziegler, E. and Susini, J., "Ion beam profiling of aspherical X-ray mirrors," Nucl. Instrum. Methods Phys. Res. A 616(2-3), 115-118 (2010).

[6] Conley, R., Shi, B., Erdmann, M., Izzo, S., Assoufid, L., Goetze, K., Mooney, T. and Lauer, K., “APS deposition facility upgrades and future plans," Adv. X-Ray/EUV Opt. Components IX 9207(Mll), C. Morawe, A. M. Khounsary, and S. Goto, Eds., 92070I (2014).

[7] Wang, T., Huang, L., Vescovi, M., Kuhne, D., Tayabaly, K., Bouet, N. and Idir, M., "Study on an effective onedimensional ion-beam figuring method," Opt. Express 27(11), 15368 (2019).

[8] Berujon, S., Wang, H., Alcock, S. and Sawhney, K., “At-wavelength metrology of hard X-ray mirror using near field speckle," Opt. Express 22(6), 6438 (2014).

[9] Wang, H., Kashyap, Y. and Sawhney, K., "Speckle based X-ray wavefront sensing with nanoradian angular sensitivity," Opt. Express 23(18), 23310 (2015).

[10] "Diamond-II Conceptual Design Report.", <https://www.diamond.ac.uk/Home/About/Vision/Diamond-II.html>. 